\title{
ON THE CANONICAL ELEMENT CONJECTURE
}

\author{
SANKAR P. DUTTA
}

\begin{abstract}
The canonical element conjecture is proved in the following two cases: (i) depth $A=\operatorname{dim} A-1, H_{m}^{n-1}(A)$ is decomposable; (ii) $\operatorname{depth} A=\operatorname{dim} A-1$, $H_{m}^{n-1}(A)^{\vee}$ is cyclic.

The equivalence of the C.E.C. and the improved new intersection theorem is also established.
\end{abstract}

In [H] M. Hochster has assigned a canonical element $\eta_{A} \in H_{m}^{n}\left(\operatorname{syz}^{n}(K)\right)$ with each $n$-dimensional local ring $(A, m, K)$ (i.e., commutative Noetherian local ring $A$ with a unique maximal ideal $m$ and $K=A / m$ ) and has conjectured that $\eta_{A} \neq 0$ for every such $A$. In elementary terms this conjecture means the following $[\mathbf{H}]$ :

For every free resolution $F$

$$
\rightarrow A^{s_{i}} \rightarrow A^{s_{i-1}} \rightarrow \cdots \rightarrow A^{s_{0}} \rightarrow K \rightarrow 0
$$

of $K$ and for every system of parameters $x_{1}, \ldots, x_{n}$ of $A$, if $\phi$ is any map of complexes $K_{*}(\mathbf{x} ; A) \rightarrow F$ which lifts the quotient surjection $A /\left(x_{1}, \ldots, x_{n}\right) \rightarrow K$, then $\phi_{n}: K_{n}(\mathbf{x} ; A) \rightarrow A^{s_{n}}$ is nonzero.

Hochster has shown that the above conjecture (henceforth C.E.C.) is equivalent to the direct summand conjecture and implies the "improved new intersection conjecture" which can be stated as follows:

ImPRoved New InTERSECTION CONJeCtURe. Let $(A, m)$ be a local ring and $F$ be a finite free complex of finitely generated free modules

$$
0 \rightarrow F_{d} \rightarrow \cdots \rightarrow F_{0} \rightarrow 0
$$

such that $H_{i}(F)$ has finite length for $i>0$ and $H_{0}(F)$ has a (nonzero) minimal generator $\nu$ such that $A \nu$ has finite length. Then $\operatorname{dim} R \leqslant d$.

It is well known that the above conjecture (henceforth I.N.I.C.) implies several other homological conjectures.

In $\$ 1$ of this paper we prove that $A$ satisfies C.E.C. $\Leftrightarrow A$ satisfies I.N.I.C. (1.4). In proving this we come across in (1.3) another equivalent version of C.E.C. which shows that actually C.E.C. boils down to understanding the relations among the resolutions of homologies of $K(\mathbf{x} ; A)$ and itself.

In $\$ 2$ we study the case when $\operatorname{depth} A=\operatorname{dim} A-1$. We prove (2.2) that $A$ satisfies C.E.C. in the following cases:

(i) when $H_{m}^{n-1}(A)^{\vee}$ is decomposable (this implies $H_{1}(\mathbf{x} ; A)$ is decomposable),

(ii) when $H_{m}^{n-1}(A)^{\vee}$ is cyclic (this implies $H_{1}(\mathbf{x} ; A)^{\vee}$ is cyclic),

Received by the editors November 18, 1985 .

1980 Mathematics Subject Classification (1985 Revision). Primary $13 \mathrm{H} 10$.

The author was supported in part by grants from the National Science Foundation.

(C)1987 American Mathematical Society $0002-9947 / 87 \$ 1.00+\$ .25$ per page 
(iii) when $\operatorname{ann}_{A} H_{m}^{n-1}(A)^{\vee} \cap\left(m-m^{2}\right) \neq \varnothing$, and

(iv) when $A$ is a U.F.D. or $\operatorname{dim} A=3$ and $H_{1}(\mathbf{x} ; A)$ is cyclic for some s.o.p. of $A$.

In 3.1 we prove the following equivalence: $A$ satisfies C.E.C. $\Leftrightarrow t \gg 0$. If $F_{*}, G_{*}$ are minimal resolutions of $A / m^{t}$ and $A / m$ respectively, and $\phi$ is any map $F_{*} \rightarrow G_{*}$ lifting the quotient surjection $A / m^{t} \rightarrow A / m$, then $\phi_{n}$ is nonzero.

By using similar arguments we remark (3.2) that for $t \gg 0$, one can make $\phi_{n+1}=\phi_{n+2}=\cdots=0$. Moreover, we also point out that there exists a natural map $k \rightarrow H_{m}^{n}\left(\operatorname{szy}^{n}(k)\right)$ which is nonzero $\Leftrightarrow$ the C.F.C. is true.

1.

1.1. Proposition. Let $F: \rightarrow A^{s_{i}} \rightarrow A^{s_{i-1}} \rightarrow \cdots \rightarrow A^{s_{0}} \rightarrow 0$ be a free complex with $H_{0}(F)=M$. Let $N \subset M$ be a submodule of $M$. Then we have a free complex $G: \rightarrow A^{d_{i}} \rightarrow A^{d_{i-1}} \rightarrow \cdots \rightarrow A^{d_{0}} \rightarrow 0$ with $H_{0}(G)=N$ and a map $\phi: G \rightarrow F$ such that the mapping cone of $\phi$ is a free resolution of $M / N$. Moreover in this case $H_{i}(G) \stackrel{\sim}{\rightarrow} H_{i}(F)$ for $i>0$.

Proof. Let $d_{0}=\mu(N)=$ minimal \# of generators of $N$. We have the following commutative diagram with horizontal rows exact:

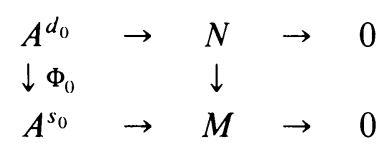

The mapping cone of

$$
\begin{array}{rrr}
0 & \rightarrow A^{d_{0}} & \rightarrow 0 \\
\downarrow & \downarrow \Phi_{0} & \\
& \stackrel{l_{1}}{\rightarrow} A^{s_{0}} & \rightarrow 0
\end{array}
$$

is $F_{1}$ :

$$
\begin{aligned}
\rightarrow & A^{s_{2}} \rightarrow A^{s_{1}} \oplus A^{d_{0}} \rightarrow A^{s_{0}} \rightarrow 0, \\
& (x, y) \rightarrow l_{1}(x)-\Phi_{0}(y) .
\end{aligned}
$$

We get $H_{i}\left(F_{1}\right)=H_{i}(F), i>1, H_{0}\left(F_{1}\right)=M / N$, and an exact sequence

$$
0 \rightarrow H_{1}(F) \rightarrow H_{1}\left(F_{1}\right) \rightarrow A^{d_{0}} \rightarrow N \rightarrow 0 .
$$

Let $d_{1}=\mu_{1}\left(H_{1}\left(F_{1}\right)\right)$. We construct $\Phi_{1}^{\prime}: A^{d_{1}} \rightarrow A^{s_{1}} \oplus A^{d_{0}}$ by sending the canonical basis of $A^{d_{1}}$ onto any set of preimages of the minimal generators of $H_{1}\left(F_{1}\right)$. The mapping cone of

$$
\begin{array}{rlrl}
0 & \rightarrow & A^{d_{1}} & \rightarrow \\
\downarrow & & \\
\downarrow \Phi_{1}^{\prime} & & \\
\cdots \rightarrow A^{s_{3}} \rightarrow A^{s_{2}} & \rightarrow & A^{s_{1}} \oplus A^{d_{0}} & \rightarrow \quad A^{s_{0}} \rightarrow 0
\end{array}
$$

is $F_{2}$ :

$$
\cdots \rightarrow A^{s_{3}} \rightarrow A^{s_{2}} \oplus A^{d_{1}} \rightarrow A^{s_{1}} \oplus A^{d_{0}} \rightarrow A^{s_{0}} \rightarrow 0 .
$$


It follows that $H_{i}\left(F_{2}\right)=H_{i}(F), i>2, H_{0}\left(F_{2}\right)=H_{0}\left(F_{1}\right), H_{1}\left(F_{2}\right)=0$, and $0 \rightarrow$ $H_{2}(F) \rightarrow H_{2}\left(F_{2}\right) \rightarrow A^{d_{1}} \rightarrow H_{1}\left(F_{1}\right) \rightarrow 0$ is exact. Continuing this way we get our complex $G$.

$$
G: \rightarrow A^{d_{1}} \rightarrow A^{d_{i-1}} \rightarrow \cdots \rightarrow A^{d_{1}} \rightarrow A^{d_{0}} \rightarrow 0
$$

such that there exists a map $\Phi: G \rightarrow F$

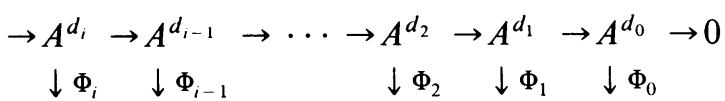

$$
\begin{aligned}
& \rightarrow A^{s_{i}} \rightarrow A^{s_{i-1}} \rightarrow \cdots \rightarrow A^{s_{2}} \rightarrow A^{s_{1}} \rightarrow A^{s_{0}} \rightarrow 0
\end{aligned}
$$

where $\Phi_{i}=p_{2} \cdot \Phi_{i}^{\prime}, p_{2}=$ projection onto the second factor, and the mapping cone of $\Phi$ is a free resolution of $M / N$.

Hence it follows that $H_{i}(F) \simeq H_{i}(G), i \geqslant 1$. Moreover if we denote by $\Psi_{i}: A^{d_{i}} \rightarrow$ $A^{d_{i-1}}$, then we have the following exact sequences:

$$
0 \rightarrow H_{i}(F) \rightarrow \operatorname{Coker} \Psi_{i+1} \rightarrow A^{d_{i-1}} \rightarrow \operatorname{Coker} \Psi_{i} \rightarrow 0
$$

for $i \geqslant 1$. Q.E.D.

1.2. In this section we are going to study the above proposition in the case when $F$ is a Koszul complex $K(\mathbf{x} ; A)$, where $\mathbf{x}=\left\{x_{1}, \ldots, x_{n}\right\}$ is a system of parameters of $A$ (x will also denote the ideal $\left(x_{1}, \ldots, x_{n}\right)$ when there is no scope of confusion). Let $H_{0}, \ldots, H_{r}$ denote the homologies of $K(\mathbf{x} ; A)$. By the above proposition we have the following diagram:

$$
\begin{aligned}
& \rightarrow A^{d_{n+1}} \rightarrow A^{d_{n}} \stackrel{\Psi_{n}}{\rightarrow} A^{d_{n-1}} \rightarrow \cdots \rightarrow A^{d_{r}} \rightarrow \cdots \rightarrow A^{d_{1}} \rightarrow 0 \\
& \downarrow \quad \downarrow g_{n} \quad \downarrow g_{n-1} \quad \downarrow g_{r} \quad \downarrow g_{1} \\
& 0 \quad \rightarrow A \stackrel{h_{n}}{\rightarrow} A^{n} \quad \rightarrow \cdots \stackrel{h_{r+1}}{\rightarrow} A^{\left({ }^{(n)}\right.} \rightarrow \cdots \stackrel{h_{2}}{\rightarrow} A^{n} \rightarrow 0
\end{aligned}
$$

where $d_{1}=\mu\left(H_{1}\right)$. We write Coker $h_{i}=G_{i-1}$ and we have $0 \rightarrow H_{1} \rightarrow G_{1} \rightarrow \mathbf{x} A \rightarrow 0$ exact.

We denote Coker $\Psi_{i+1}$ by $H_{i}^{\prime}$ and the vertical maps $A^{d_{i}} \rightarrow A^{\left(i_{i}\right)}$ by $g_{i}$.

From the above diagram we obtain a smaller diagram with both the horizontal complexes representing minimal resolutions of $H_{r}^{\prime}$ and $G_{r}^{\prime}$ respectively:

$$
\begin{aligned}
& A^{d_{n+1}} \stackrel{\Psi_{n+1}}{\rightarrow} A^{d_{n}} \rightarrow A^{d_{n-1}} \rightarrow \cdots \quad \rightarrow \quad A^{d_{r}} \quad \rightarrow H_{r}^{\prime} \rightarrow 0
\end{aligned}
$$

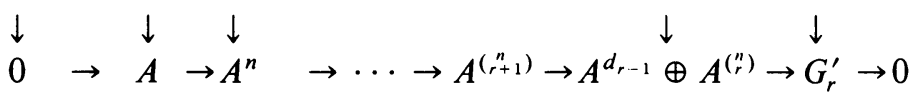

where $G_{r}^{\prime}=G_{r} \oplus A^{d_{r-1}}$. We also obtain from the above diagram:

$$
\begin{array}{r}
0 \rightarrow H_{i} \rightarrow H_{i}^{\prime} \rightarrow A^{d_{i-1}} \rightarrow H_{i-1}^{\prime} \rightarrow 0 \\
\quad \cdots+\cdots \cdots+H_{2} \rightarrow H_{2}^{\prime} \rightarrow A^{d_{1}} \rightarrow H_{1} \rightarrow 0
\end{array}
$$


Fact 1. Since the $H_{i}$ 's are modules of finite length, the $\operatorname{Ext}^{j}\left(H_{i}^{\prime}, A\right)$ are also. (This follows from (3).) (We do not use any suffixes for the ring when we take ext over $A$.)

Fact 2. Let $\operatorname{Im} g_{n}=A$; then we can get a basis $e_{1}, \ldots, e_{d_{n}}$ of $A^{d_{n}}$ such that $g_{n}\left(e_{i}\right)=1, g_{n}\left(e_{1}\right)=0, i=2, \ldots, d_{n}$. Hence from (2) we get $\operatorname{Im}\left(\Psi_{n+1}\right) \subset$ submodule generated by $e_{2}, \ldots, e_{d_{n}}$.

Hence $\operatorname{syz}^{n-r}\left(H_{r}^{\prime}\right)$ has a free summand and we can write

$$
\operatorname{syz}^{n-r}\left(H_{r}^{\prime}\right)=A \oplus S_{n-r}^{\prime} .
$$

Notation. If $M$ is finitely generated and $A^{r_{1}} \stackrel{\theta}{\rightarrow} A^{r_{0}} \rightarrow M$ is exact, then we write $D(M)=\operatorname{Coker} \Theta^{*}$.

When $\operatorname{Im} g_{n}=A$ from (2) we see that $D s_{n-r-1}\left(H_{r}^{\prime}\right)$ has a generator which is killed by the ideal $\left(x_{1}, \ldots, x_{n}\right)$.

1.3. Theorem. A satisfies C.E.C. $\Leftrightarrow \operatorname{Im} g_{n} \neq A$.

Proof. $\Rightarrow$ : If possible let $\operatorname{Im} g_{n}=A$. By applying $\operatorname{Hom}(-, A)$ to the top complex in (1) we get

$$
0 \rightarrow A^{d_{1}^{*}} \rightarrow A^{d_{2}^{*}} \rightarrow \cdots \rightarrow A^{d_{n-2}^{*}} \stackrel{\Psi_{n-1}^{*}}{\rightarrow} A^{d_{n-1}^{*}} \rightarrow 0
$$

By Fact 2 all homologies, except the zeroth one, have finite length and Coker $\Psi_{n-1}^{*}$ $=D s_{n-r-1}\left(H_{r}^{\prime}\right)$. Since $D s_{n-r-1}\left(H_{r}^{\prime}\right)$ has a generator killed by $\left(x_{1}, \ldots, x_{n}\right)$ we arrive at a contradiction (because C.E.C. $\Rightarrow$ I.N.I.C. $[$ H]). Q.E.D.

$\Leftarrow$ : Suppose $A$ does not satisfy C.E.C. Then we have the following situation:

$$
\begin{aligned}
& 0 \rightarrow A \rightarrow A^{n} \rightarrow \cdots \rightarrow A^{\left(\begin{array}{c}
n \\
r
\end{array}\right)} \rightarrow \cdots \rightarrow A^{n} \rightarrow A \rightarrow A / \mathbf{x} \rightarrow 0 \\
& \cdots \rightarrow A^{t_{n}} \stackrel{\theta_{n}}{\rightarrow} A^{t_{n-1}} \rightarrow \cdots \rightarrow A^{t_{r}} \rightarrow \cdots \rightarrow A^{t_{1}} \rightarrow A \rightarrow A / m \rightarrow 0
\end{aligned}
$$

where the bottom row is a minimal resolution of $A / m$. We write $-*=\operatorname{Hom}(-, A)$

Applying $\operatorname{Hom}(-, A)$ to the above diagram we get the following commutative diagram:

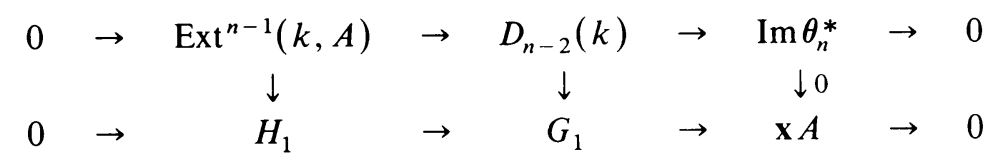

Hence we have

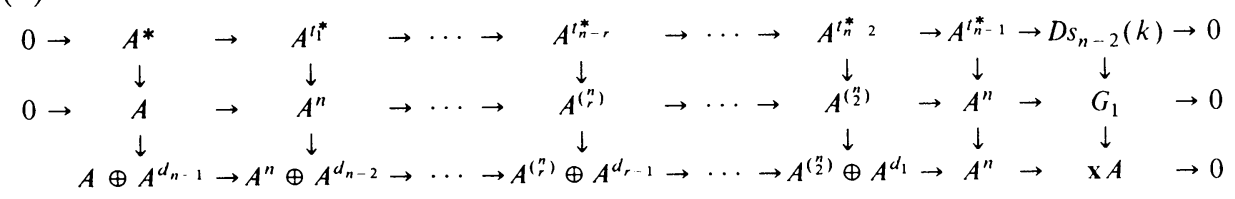

(Recall, the resolution of $\mathbf{x} A$ is obtained by taking the mapping cone of (1).) 
Since by (4) the composite map $D s_{n-2}(k) \rightarrow G_{1} \rightarrow \mathbf{x} A$ is zero, the resulting maps in (5) are homotopic to zero. So we get a family of homotopy maps $\left\{\mu_{i}\right\}$. But now from

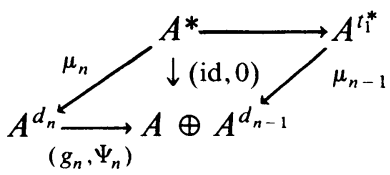

it follows that $\operatorname{Im} g_{n}=A$, which is a contradiction. Q.E.D.

1.4. Corollary. C.E.C. is true $\Leftrightarrow$ I.N.I.C. is true.

Proof. $\Rightarrow:$ See $[\mathbf{H}]$.

$\Leftarrow$ : I.N.I.C. $\Rightarrow \operatorname{Im} g_{n} \neq A \Rightarrow$ C.E.C.

RemarK. (i) To be more precise, C.E.C. holds $\Rightarrow \operatorname{Im} g_{n} \neq$ a principal ideal in $A$.

(ii) It is easy to see that $\operatorname{Im} g_{n} \neq A$ is equivalent to the criteria pointed out by $\mathrm{P}$. Roberts [R].

2. In this section we assume depth $A=\operatorname{dim} A-1$. Let $n$ be the dimension of $A$.

2.1. Let

$$
\rightarrow A^{r_{n-1}} \rightarrow A^{r_{n-2}} \rightarrow \cdots \rightarrow A^{r_{0}} \rightarrow H_{1} \rightarrow 0
$$

be a minimal resolution of $H_{1}$.

We have the following diagram:

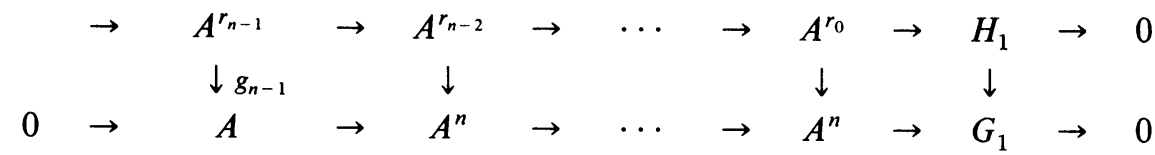

By Theorem (1.3) C.E.C. holds in $A \Leftrightarrow \operatorname{Im} g_{n-1} \neq A$. As described in $\S 1.2$, Fact 2, if $\operatorname{Im} g_{n-1}=A$, then $\operatorname{syz}^{n-1}\left(H_{1}\right)$ has a free direct summand and moreover $D s_{n-2}\left(H_{1}\right)$ has a generator $\nu$ killed by $\left(x_{1}, \ldots, x_{n}\right)$. Actually this generator is killed by $I$, where $I=\operatorname{ann}_{A} H_{1}$, since it is a generator for $\operatorname{Ext}^{n-1}\left(H_{1}, A\right)$ too. Let $J \subseteq I$ be any ideal. We have the following commutative diagram of exact sequences:

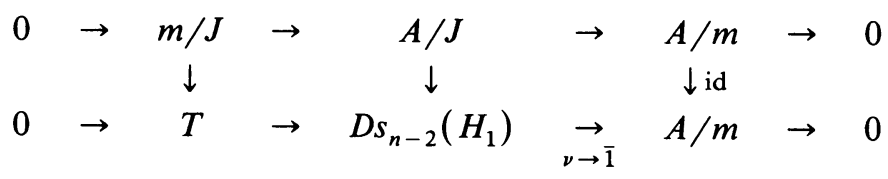

where the map in the middle is obtained by sending 1 to the generator $\nu$ of $D s_{n-2}\left(H_{1}\right)$ which is the image of $A / \mathbf{x}$ obtained from (6) by applying $\operatorname{Hom}(-, A)$ to (6). Since $D s_{n-2}\left(H_{1}\right)$ has projective dimension $n-1$, by applying $\operatorname{Hom}(-, A)$ to the above diagram we get

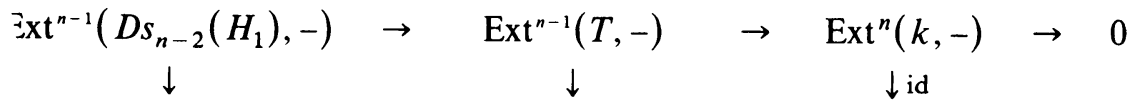

$$
\begin{aligned}
& \operatorname{Ext}^{n-1}(A / J,-) \quad \rightarrow \quad \operatorname{Ext}^{n-1}(m / J,-) \stackrel{\delta}{\rightarrow} \operatorname{Ext}^{n}(k,-)
\end{aligned}
$$


and

$$
\begin{aligned}
& \begin{array}{ccc}
\operatorname{Ext}^{n+i}(T,-) & \simeq \operatorname{Ext}^{n+i+1}(k, A), \quad i \geqslant 0 \\
\downarrow \text { id } &
\end{array} \\
& \operatorname{Ext}^{n+i}(A / I,-) \quad \rightarrow \quad \operatorname{Ext}^{n+i}(m / I, A) \stackrel{\delta_{i}}{\rightarrow} \quad \operatorname{Ext}^{n+i+1}(k, A)
\end{aligned}
$$

This implies that $\delta$ is onto and the $\delta_{i}$ 's are onto and they split.

Hence all the maps

$$
\operatorname{Ext}^{n+i}(k,-) \rightarrow \operatorname{Ext}^{n+i}(A / J,-)
$$

are zero maps and

$$
\operatorname{Ext}^{n+i}(m / J,-) \stackrel{\sim}{\rightarrow} \operatorname{Ext}^{n+i}(A / J,-) \oplus \operatorname{Ext}^{n+i+1}(k,-) \text { for } i \geqslant 0 .
$$

Thus we have proved the following.

Proposition. If $\operatorname{dim} A=n, \operatorname{depth} A=n-1$, and $A$ does not satisfy C.E.C., then for any ideal $J \subset I=\operatorname{ann}_{A} H_{1}(\mathbf{x} ; A)$ and for any module $M$ the map

$$
\operatorname{Ext}^{n+i}(k, M) \rightarrow \operatorname{Ext}^{n+i}(A / J, M)
$$

is a zero-map and

$$
\operatorname{Ext}^{n+i}(m / J, M) \rightarrow \operatorname{Ext}^{n+i}(A / J, M) \oplus \operatorname{Ext}^{n+i+1}(k, M)
$$

is an isomorphism for every $i>0$.

2.2. Theorem. Let $(A, m, k)$ be a local ring with $\operatorname{dim} A=n$ and $\operatorname{depth} A=n-1$. Then $A$ satisfies $C$.E.C. in the following cases:

(i) if $H_{m}^{n-1}(A)$ is decomposable,

(ii) if $H_{m}^{n-1}(A)^{\vee}$ is cyclic,

(iii) if Ann $H_{m}^{n-1}(A) \cap\left(m-m^{2}\right) \neq \varnothing$, and

(iv) if $A$ is U.F.D. or $\operatorname{dim} A=3$ and $H_{1}(\mathbf{x} ; A)$ is cyclic for some s.o.p. of $A$.

Proof. Without any loss of generality we can assume $A$ is complete and hence $A=R / I$, where $R$ is a complete intersection with $\operatorname{dim} R=\operatorname{dim} A$. We can also choose $x_{1}, \ldots, x_{n}$ in such a way that $x_{1}, \ldots, x_{n-1}$ is an $A$-sequence and $H_{1}=$ $\left(0: x_{n}\right)_{\bar{A}}=H_{m}^{0}(\bar{A})$, where $\bar{A}=A /\left(x_{1}, \ldots, x_{n-1}\right)$.

Hence $H_{1}=H_{m}^{0}(\bar{A})=\operatorname{Ext}^{1}(A, R) /\left(x_{1}, \ldots, x_{n-1}\right) \operatorname{Ext}^{1}(A, R)$. We know by local duality that $H_{m}^{n-1}(A)^{\vee} \simeq \operatorname{Ext}^{1}(A, R)$.

(i) As in the proof of Theorem (1.3), if C.E.C. does not hold we have the following commutative diagram:

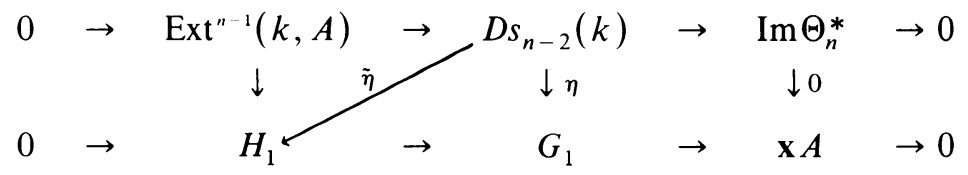

$\operatorname{Im} \theta_{n}^{*} \stackrel{0}{\rightarrow} \mathbf{x} A$ implies $\eta$ factors through $\tilde{\eta}$. Hence $\operatorname{Ext}^{n-1}\left(H_{1}\right) \rightarrow \operatorname{Ext}^{n-1}\left(D s_{n-2}(k), A\right)$ $=k$ is onto. We have, by what has been discussed already in this section, $H_{1}$ is decomposable whenever $H_{m}^{n-1}(A)$ is. Now if we can show that the injection 
$\operatorname{Ext}^{n-1}(k, A) \stackrel{i}{\rightarrow} D s_{n-2}(k)$ induces a nonzero map

$$
i^{*}: \operatorname{Ext}^{n-1}\left(D s_{n-2}(k), A\right) \rightarrow \operatorname{Ext}^{n-1}\left(\operatorname{Ext}^{n-1}(k, A), A\right),
$$

then it will follow from the commutative diagram

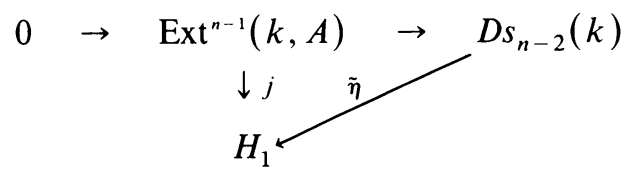

that the image of the map

$$
\begin{aligned}
j^{*}: \operatorname{Hom}\left(H_{1}, H_{1}\right) & =\operatorname{Ext}^{n-1}\left(H_{1}, A\right) \rightarrow \operatorname{Ext}^{n-1}\left(\operatorname{Ext}^{n-1}(k, A), A\right) \\
& =\operatorname{Hom}\left((0: m) H_{1},(0: m) H_{1}\right)
\end{aligned}
$$

is just $k$. But if $H_{1}$ is decomposable, this leads to a contradiction. Thus C.E.C. must hold when $H_{m}^{n-1}(A)$ is decomposable. Q.E.D.

LEMMA. The exact sequence

$$
0 \rightarrow \operatorname{Ext}^{n-1}(k, A) \stackrel{i}{\rightarrow} D s_{n-2}(k) \rightarrow \operatorname{Im} \Theta_{n}^{*} \rightarrow 0
$$

induces a nonzero map

$$
i^{*}: k=\operatorname{Ext}^{n-1}\left(D s_{n-2}(k), A\right) \rightarrow \operatorname{Ext}^{n-1}\left(\operatorname{Ext}^{n-1}(k, A), A\right) .
$$

Proof. If possible let the above map be zero. We have the following diagram:

$$
\begin{aligned}
& \rightarrow A^{d_{n}} \rightarrow A^{d_{n-1}} \rightarrow A^{d_{n-2}} \rightarrow \cdots \rightarrow A^{d_{1}} \rightarrow A^{d_{0}} \rightarrow \operatorname{Ext}^{n-1}(k, A) \rightarrow 0
\end{aligned}
$$

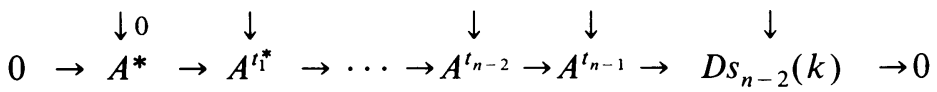

where the top row is a minimal resolution of $\operatorname{Ext}^{n-1}(k, A)$. Since $i^{*}$ is 0 , we can take the map $A^{d_{n-1}} \rightarrow A^{*}$ in the above diagram as a 0 -map. Applying $\operatorname{Hom}(-, A)$ we get

$$
\begin{aligned}
& \rightarrow A^{t_{n}} \rightarrow A^{t_{n-1}} \rightarrow \cdots \rightarrow A^{t_{1}} \rightarrow A \rightarrow \quad \rightarrow \quad \rightarrow 0 \\
& 0 \stackrel{\downarrow}{\downarrow} A^{d_{0}^{*}} \rightarrow \cdots \rightarrow A^{\stackrel{\downarrow}{d_{n-2}^{*}} \rightarrow A^{d_{n-1}^{*}}} \rightarrow D s_{n-2}\left(\operatorname{Ext}^{\downarrow 0}(k, A)\right)
\end{aligned}
$$

since both rows are exact and the vertical maps lift $k \stackrel{0}{\rightarrow} D s_{n-2}\left(\operatorname{Ext}^{n-1}(k, A)\right)$, we see by applying $\operatorname{Hom}(-, A)$ to the above diagram that $i=0: \operatorname{Ext}^{n}(k, A) \rightarrow D s_{n-2}(k)$, which is a contradiction. Q.E.D.

(ii) Since $H_{m}^{n-1}(A)^{\vee}$ is cyclic, it follows that $H_{1}^{\vee}$ is cyclic and hence $H_{1}^{\vee} \simeq A / I$. Suppose C.E.C. does not hold in this case. Then from (2.1) we get the exact sequence

$$
0 \rightarrow \operatorname{Ext}^{n-1}(k, A) \rightarrow \operatorname{Ext}^{n-1}(A / I, A) \rightarrow \operatorname{Ext}^{n-1}(m / I, A) \rightarrow \operatorname{Ext}^{n}(k, A) \rightarrow 0 .
$$

But

$$
\begin{aligned}
& \operatorname{Ext}^{n-1}(k, A)=\operatorname{Hom}\left(k, H_{1}\right)=A / m, \\
& \operatorname{Ext}^{n-1}(A / I, A)=\operatorname{Hom}\left(A / I, H_{1}\right)=(A / I),
\end{aligned}
$$

and

$$
\operatorname{Ext}^{n-1}(m / I, A)=\operatorname{Hom}\left(m / I, H_{1}\right)=(m / I) .
$$


Since $0 \rightarrow m / I \rightarrow A / I \rightarrow A / m \rightarrow 0$ is exact, this implies $\operatorname{Ext}^{n}(k, A)=0$, which is a contradiction. Q.E.D.

Note. When C.E.C. does not hold we have

$$
\begin{array}{ccc}
\operatorname{Ext}^{n}(k, A) & \stackrel{0}{\rightarrow} & \operatorname{Ext}^{n}(A / I, A) \\
\| & & \| \\
\operatorname{Ext}_{\bar{A}}^{1}(k, \bar{A}) & \stackrel{0}{\rightarrow} & \operatorname{Ext}^{1}(A / I, \bar{A})
\end{array}
$$

where $\bar{A}=A /\left(x_{1}, \ldots, x_{n-1}\right)$. Now from $0 \rightarrow H_{1} \rightarrow \bar{A} \rightarrow x_{n} \bar{A} \rightarrow 0$ we get $0 \rightarrow$ $\operatorname{Ext}_{\bar{A}}^{1}\left(k, H_{1}\right) \rightarrow \operatorname{Ext}_{\bar{A}}^{1}(k, \bar{A})$, here $H_{1}=H_{m}^{0}(\bar{A})$. Since $l\left(\operatorname{Ext}_{\bar{A}}^{1}\left(k, H_{1}\right)\right)=$ $l\left(\operatorname{Tor}_{1}^{\bar{A}}\left(k, H_{1}^{\vee}\right)\right.$, when C.E.C. does not hold, $\operatorname{Ext}_{\bar{A}}^{1}\left(k, H_{1}\right) \simeq \operatorname{Ext}_{\bar{A}}^{1}(k, \bar{A})$. Hence when C.E.C. does not hold we get $\operatorname{Tor}_{1}^{\bar{A}}\left(\bar{A} / I, H_{1}^{\vee}\right) \stackrel{0}{\rightarrow} \operatorname{Tor}_{1}^{\bar{A}}\left(k, H_{1}^{\vee}\right)$.

(iii) In (ii) we have seen that if C.E.C. does not hold then the map

$$
\operatorname{Tor}_{1}^{\bar{A}}\left(\bar{A} / I, H_{1}^{\vee}\right) \rightarrow \operatorname{Tor}_{1}^{\bar{A}}\left(k, H_{1}^{\vee}\right)
$$

is 0 ; here $\bar{A}=A\left(x_{1}, \ldots, x_{n-1}\right), I=\operatorname{Ann}_{A} H_{1}$. This implies $H_{1}^{\vee} \otimes m \simeq H_{1}^{\vee} \otimes m / I$, which is impossible if $I \cap\left(m-m^{2}\right) \neq \varnothing$.

(iv) If $A$ is a U.F.D., then $H_{1}(\mathbf{x} ; A) \simeq H_{1}(\mathbf{x} ; A)^{\vee}$ (one may have to choose $\mathbf{x}$ a bit cautiously). Hence, when $H_{1}(\mathbf{x} ; A)$ is cyclic, we are done by (ii).

When $\operatorname{dim} A=3$. In this case $l\left(\operatorname{Ext}^{1}(A, R)\right)<\infty$. This allows us to choose an s.o.p. $\left\{x_{1}, x_{2}, x_{3}\right\}$ (and hence all its powers) contained in $\operatorname{Ann}_{A} \operatorname{Ext}^{1}(A, R)$.

We consider the exact sequence

$$
0 \rightarrow I \rightarrow R \rightarrow A \rightarrow 0
$$

Applying $\operatorname{Hom}(-, R)$ we get

$$
0 \rightarrow A^{*} \rightarrow R \rightarrow I^{*} \rightarrow \operatorname{Ext}_{R}^{1}(A, R) \rightarrow 0 .
$$

Applying $\otimes R /\left(x_{1}, x_{2}\right)$ we get

$$
0 \rightarrow \operatorname{Ext}_{R}^{1}(A, R) \rightarrow A^{*} /\left(x_{1}, x_{2}\right) A^{*} \rightarrow D \rightarrow 0, \quad \operatorname{depth} D>0 .
$$

Hence $\operatorname{Ext}_{A}^{2}\left(k, A^{*}\right)=\operatorname{Hom}\left(k, \operatorname{Ext}_{R}^{1}(A, R)\right)=\operatorname{Hom}\left(H_{1} / m H_{1}, E\right)$. If C.E.C. does not hold, then by the same method as used in (ii) we get $\operatorname{Tor}_{1}^{\bar{A}}\left(\bar{A} / I, H_{1}\right) \rightarrow \operatorname{Tor}_{1}^{\bar{A}}\left(k, H_{1}\right)$ to be 0 . But when $H_{1}$ is cyclic, $H_{1} \simeq \bar{A} / I$ and hence this is impossible.

3.

3.1. Theorem. A satisfies C.E.C. $\Leftrightarrow$ for $t \gg 0$, given $F_{*}, G_{*}$, minimal resolutions of $A / m^{t}$ and $A / m$ respectively, and $\phi$ any map $F_{*} \rightarrow G_{*}$ lifting the quotient surjection $A / m^{t} \rightarrow A / m$, then $\phi_{n}$ is nonzero.

Proof. $\Leftarrow:$ This is obvious, for if $\phi_{n}$ is zero, one can choose an s.o.p. $\left\{x_{1}, \ldots, x_{n}\right\}$ $\subset m^{t}$ and arrive at a contradiction.

$\Leftarrow$ : Let $S=\operatorname{syz}^{n}(k)$ in a minimal resolution of $k$. If possible, let $\eta_{A}=0$. Since $H_{m}^{n}(S)=\lim _{\rightarrow} \operatorname{Ext}^{n}\left(A / m^{t}, S\right), \eta_{A}$ actually becomes 0 in $\operatorname{Ext}^{n}\left(A / m^{t}, s\right)$ for $t \gg 0$. 
Let $T=\operatorname{syz}^{n}\left(A / m^{t}\right)$ in a minimal resolution of $A / m^{t}$. We consider the diagram

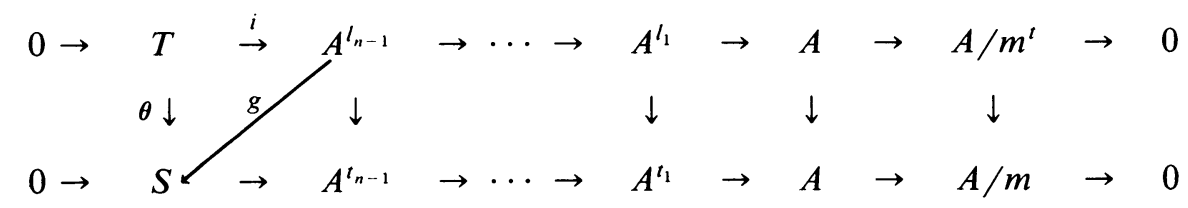

where the upper and lower rows are obtained from minimal resolutions $F_{*}, G_{*}$ of $A / m^{t}$ and $A / m$ respectively. By what has been stated above it follows that $\exists g: A^{l_{n-1}} \rightarrow S$ such that $g \cdot i=\theta$. This implies that, without any loss of generality, we can take $\theta$ to be the 0 -map. Hence we arrive at a contradiction.

3.2. Remarks. (i) We write $S_{i}=\operatorname{syz}^{i}(k)$ in a minimum resolution of $k$. Since $\operatorname{Ext}^{n+1}\left(k, S_{n+1}\right) \rightarrow H_{m}^{n+1}\left(S_{n+1}\right)=0$, it follows that for $t \gg 0$, for any lift $\phi: F_{*} \rightarrow$ $G_{*}$ of the canonical surjection $A / m^{t} \rightarrow A / m, 0=\phi_{n+1}=\phi_{n+2}=\cdots$.

(ii) We have the exact sequences

$$
\begin{aligned}
& 0 \rightarrow S_{n} \rightarrow A^{t_{n-1}} \rightarrow S_{n-1} \rightarrow 0, \ldots, \\
& 0 \rightarrow S_{i} \rightarrow A^{t_{i-1}} \rightarrow S_{i-1} \rightarrow 0, \ldots, 0 \rightarrow S_{1} \rightarrow A \rightarrow k \rightarrow 0
\end{aligned}
$$

which give rise to the following commutative diagram:

$$
\begin{aligned}
& k \quad \rightarrow \operatorname{Ext}^{1}\left(k, S_{1}\right) \rightarrow \operatorname{Ext}^{2}\left(k, S_{2}\right) \rightarrow \cdots \rightarrow \operatorname{Ext}^{n-1}\left(k, S_{n-1}\right) \rightarrow \operatorname{Ext}^{n}\left(k, S_{n}\right)
\end{aligned}
$$

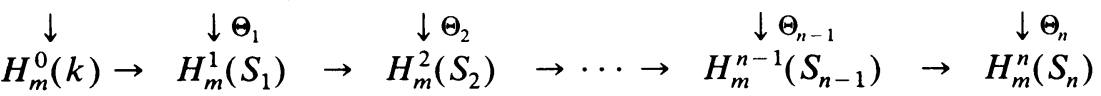

One can check that $n_{A}$ is nothing but the image of $k$ in this long commutative diagram. Moreover it can also be shown that $\Theta_{i}, 1 \leqslant i \leqslant n-1$, takes Id $\in$ $\operatorname{Hom}\left(S_{i}, S_{i}\right)$ to a nonzero element in $H_{m}^{i}\left(S_{i}\right)$.

In other words the canonical element $\eta_{A}=\eta_{A}^{n}$ corresponding to $S_{n}$ is the natural image of canonical elements $\eta_{A}^{i}=\Theta^{i}$ [image of $\operatorname{Id} \in \operatorname{Hom}\left(S_{i}, S_{i}\right)$ in $\operatorname{Ext}^{i}\left(k, S_{i}\right)$ ] corresponding to $S_{i}$ for $0 \leqslant i \leqslant n-1$. Thus $\eta_{A}$ is nothing but the image of $k \rightarrow H_{m}^{n}\left(S_{n}\right)$ in a very natural way-the conjecture asserts that this map is nonzero.

\section{REFERENCES}

$[\mathbf{H}] \mathrm{M}$. Hochster, Canonical elements in local cohomology modules and the direct summand conjecture, $\mathrm{J}$. Algebra 84 (1983), 503-553.

[R] P. Roberts, The equivalence of two forms of the canonical element conjecture, preprint.

Department of Mathematics, University of Pennsylvania, Philadelphia Pennsylvania 19104

Current address: Department of Mathematics, University of Illinois, Urbana, Illinois 61801 